\title{
Identifying targets for COPD treatment through gene expression analyses
}

\author{
Zhi-Hua Chen' \\ Hong Pyo Kim' \\ Stefan W Ryter' \\ Augustine MK Choi ${ }^{2}$ \\ 'Division of Pulmonary, Allergy and \\ Critical Care Medicine, Department \\ of Medicine, University of Pittsburgh, \\ Pittsburgh, PA, USA; ${ }^{2}$ Pulmonary and \\ Critical Care Medicine, Brigham and \\ Women's Hospital, Harvard Medical \\ School, Boston, MA, USA
}

\begin{abstract}
Despite the status of chronic obstructive pulmonary disease (COPD) as a major global health problem, no currently available therapies can limit COPD progression. Therefore, an urgent need exists for the development of new and effective treatments for COPD. An improved understanding in the molecular pathogenesis of COPD can potentially identify molecular targets to facilitate the development of new therapeutic modalities. Among the best approaches for understanding the molecular basis of COPD include gene expression profiling techniques, such as serial analysis of gene expression or microarrays. Using these methods, recent studies have mapped comparative gene expression profiles of lung tissues from patients with different stages of COPD relative to healthy smokers or non-smokers. Such studies have revealed a number of differentially-regulated genes associated with COPD progression, which include genes involved in the regulation of inflammation, extracellular matrix, cytokines, chemokines, apoptosis, and stress responses. These studies have shed new light on the molecular mechanisms of COPD, and suggest novel targets for clinical treatments.
\end{abstract}

Keywords: COPD, gene expression, therapeutic targets

\section{Introduction}

Chronic obstructive pulmonary disease (COPD) is a major global health problem predicted to rank as the third greatest cause of mortality by the end of the next decade (Lopez and Murray 1998). COPD is a slowly progressive and poorly reversible inflammatory disease characterized by the functional abnormality of airway obstruction, which includes emphysema, chronic bronchitis, and small airways disease (Pauwels et al 2001). Cigarette smoking is the most important risk factor for the development of COPD. However, only $10 \%-15 \%$ of smokers develop the disease, implying genetic factors may increase the risk in certain individuals (Mayer and Newman 2001). Smoking cessation is the most efficient therapeutic intervention so far shown to reduce the disease progression (Barnes 1999, 2004). However, the poor long-term success rate of smoking cessation ( $16 \%$ at 6 months) requires the development of still more effective approaches (Barnes 2004). A number of experimental therapeutics have been studied for their potential in COPD treatment or prevention, which include antioxidants, antiinflammatory agents, and/or small molecule inhibitors of proinflammatory signaling pathways, thus far with limited success (Barnes and Stockley 2005).

The lack of drug development for this disease is due to the relatively recent emergence of research addressing the molecular and cellular basis of COPD (Barnes et al 2003; Barnes 2005; Barnes and Hansel 2004; Donnelly and Barnes 2006; Yoshida and Tuder 2006). A general scheme of the molecular pathways involved in COPD pathogenesis and potential areas for therapeutic intervention is shown in Figure 1.

Gene expression profiling of human diseased tissues may provide insights into the molecular mechanisms of human disease and may eventually lead to the identification of novel therapeutic targets. The goal of this article is to summarize recent gene 


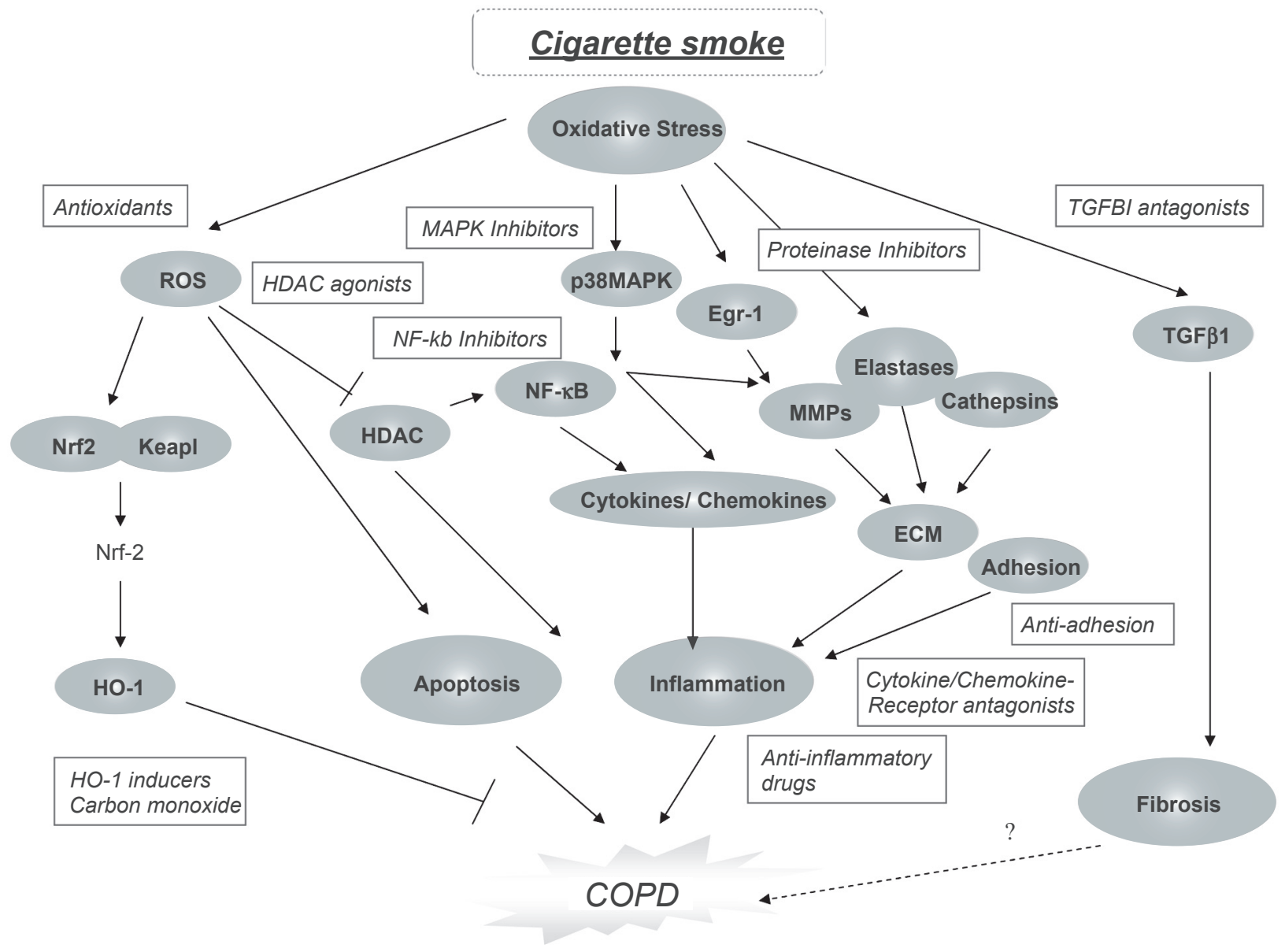

Figure I Molecular Mechanisms in COPD pathogenesis and potential therapeutic targets. A number of signaling pathway components have been identified which regulate apoptosis, inflammation and fibrosis potentially associated with COPD pathogenesis. Many of these have been identified as possible targets for therapeutic intervention using small molecule inhibitors or antagonists.

Abbreviations: COPD, chronic obstructive pulmonary disease; ECM, extracellular matrix; Egr-I, early growth response-I; HDAC, histone deacetylase; HO-I, heme oxygenase-I; Keap-I, Kelch-like ECH associating protein- I; MMP, matrix metalloproteinase; NF- $\kappa B$, nuclear factor-kappa-B; Nrf-2, nuclear factor-E2-related factor-2; p38 MAPK, p38 mitogenactivated protein kinase; ROS, reactive oxygen species; TGF $\beta$ I, transforming growth factor-B I.

expression profiling studies in COPD, and to integrate the findings with biochemical observations in COPD/emphysema pathogenesis and drug development.

\section{Gene expression profiling studies in emphysema/COPD}

Recent studies have analyzed lung gene expression profiles of COPD patients at different stages of disease progression relative to control subjects (Golpon et al 2004; Ning et al 2004; Spira et al 2004; Pierrou et al 2007; Wang et al 2008). Two general approaches have been applied with respect to sample generation: the analysis of total lung homogenates and the analysis of discrete cell populations. While both approaches are valid, they each have some limitations. Analysis of whole lung homogenates provides a global view of lung gene expression profiles, but due to the heterogeneous nature of the lung, signals originating in one cell type may be diluted by cell populations which do not respond in the same way. Furthermore, signals specific to specialized or rare cell populations may not be detectable. Detectable increases in gene expression can be localized to specific cell types by subsequent validation with real-time polymerase chain reaction (RT-PCR) or immunohistochemical approaches. The analysis of discrete cell populations by microarray will provide a clearer understanding of the response in individual cell types, but at the same time may overlook significant responses of the lung as a whole. The latter approach is technically more challenging, and requires the preparation of relatively pure samples, sometimes requiring advanced techniques such as laser capture microdissection.

Golpon and colleagues (2004) characterized differential gene expression in total lung homogenates between advanced emphysema and healthy human subjects, and also compared "usual" emphysema with $\alpha_{1}$-antitrypsin $\left(\alpha_{1}\right.$-AT) deficiency, 
the genetic cause of emphysema. Lungs with severe emphysema displayed decreases in overall global gene expression, with pronounced increases in transcripts encoding proinflammatory mediators, immune-response proteins, and proteolytic enzymes. Many of these differentially-expressed genes were also observed in $\alpha_{1}$-AT lung, which displayed an additional set of differentially-regulated genes involved in protein synthesis, energy metabolism, and immune function. The authors concluded that the two types of emphysema can be distinguished from each other, and from normal tissue by gene expression profiles (Golpon et al 2004).

Ning and colleagues (2004) analyzed gene expression profiles in total lung tissue obtained from COPD patients (GOLD2) that were smokers vs GOLD0 smokers, using serial analysis of gene expression (SAGE) and comparative microarray analysis. Analysis of the same RNA sample set revealed 327/261 differentially-expressed transcripts by SAGE or microarray, respectively. The genes differentially expressed in GOLD2 vs GOLD0 were identified as belonging to several functional classes, including transcription factors, growth factors, cytokines, chemokines, and extracellular matrix (ECM) proteins. For example, analysis of GOLD2 smoker's lungs tissue revealed upregulation of transcripts for pro-inflammatory mediators, (eg, TGF $\beta-1$, CX3CL1, CTGF, CYR61, TNFSF10, and the interleukin-1 receptor), relative to GOLD0 smokers. Additionally, Egr-1 was identified as a major pro-inflammatory transcriptional regulator that was upregulated in GOLD2 vs GOLD0 lung tissue. On the other hand, lung tissue from GOLD0 smokers displayed higher levels of expression of transcripts encoding ECM proteins (eg, collagens), relative to GOLD2 smokers. This study reported a high level of agreement between SAGE and microarray analyses of the same sample set, in particular with respect to the expression of inflammatory mediators and ECM proteins (Ning et al 2004).

Using high-density microarrays, Spira and colleagues (2004) evaluated gene expression in lung tissues from patients with severe emphysema, removed during lung reduction surgery, relative to tissues from patients with mild emphysema undergoing surgical resection of lung nodules, or normal smokers. From this analysis, 102 genes were identified whose differential expression distinguished severe emphysema from mildly emphysematous or normal tissue. This analysis revealed the upregulation of many ECM-related proteins and the downregulation of immune-function and cell signaling-related genes in severe vs mild emphysema (Spira et al 2004). Furthermore, this study identified subsets of genes whose expression were positively or negatively correlated with decline of lung functional parameters (Spira et al 2004).

A more recent study compared surgically resected lung tissue from nonsmokers with lung tissues from COPD patients at GOLD stages 0-3 (Wang et al 2008). Morphometric analysis of samples revealed a subset of genes whose expression varied with the sample composition $(\%$ parenchyma). Furthermore 203 genes were identified whose expression correlated to lung function. This analysis revealed the upregulation of ECM-related genes and apoptosis genes, and the downregulation of anti-inflammatory genes in COPD tissues (Wang et al 2008).

Pierrou and colleagues (2007) evaluated differential gene expression in bronchial epithelial cell brushings taken from COPD smokers, healthy smokers and healthy nonsmokers, with a microarray analysis focused on oxidative stress genes. A large number of genes involved in oxidative stress responses were differentially expressed by smoking relative to nonsmoking controls, with metallothioneins prominently expressed. An additional set of genes were differentially regulated in COPD patients relative to healthy smokers. These included thioredoxins, superoxide dismutase-2, and genes involved in energy metabolism, respiration, and apoptosis. Analysis of both comparisons (COPD smokers vs healthy smokers, and healthy smokers vs nonsmokers) revealed a common set of upregulated genes that included cytochrome p450 isozymes and aldo-keto reductases. Among those genes downregulated in healthy smokers, but upregulated at an early stage of COPD (GOLD0-1), a trend toward decreased expression with increasing disease severity was noted for the later stages of COPD (GOLD2-4). Microarray analysis was also performed on primary human bronchial epithelial cells derived from the same subjects and exposed to in vitro cigarette smoke, and these results were confirmatory of those obtained from healthy smokers vs nonsmokers (Pierrou et al 2007).

There was little apparent overlap between specific gene sets between the various studies. However some similarities were observed in the functional categories of differentially expressed genes, with variations in ECM related proteins and inflammatory regulators the most common (Table 1).

A primary difference between the human gene expression profiling studies was the use of epithelial cell brushings in the study of Pierrou and colleagues (2007), vs total lung tissue in the studies of Spira (2004); Ning (2004); and Wang (2008) and their colleagues. Wang (2008) represented a much more heterogeneous lung cell population. The studies by Wang and colleagues (2008) demonstrated that variations in sample 


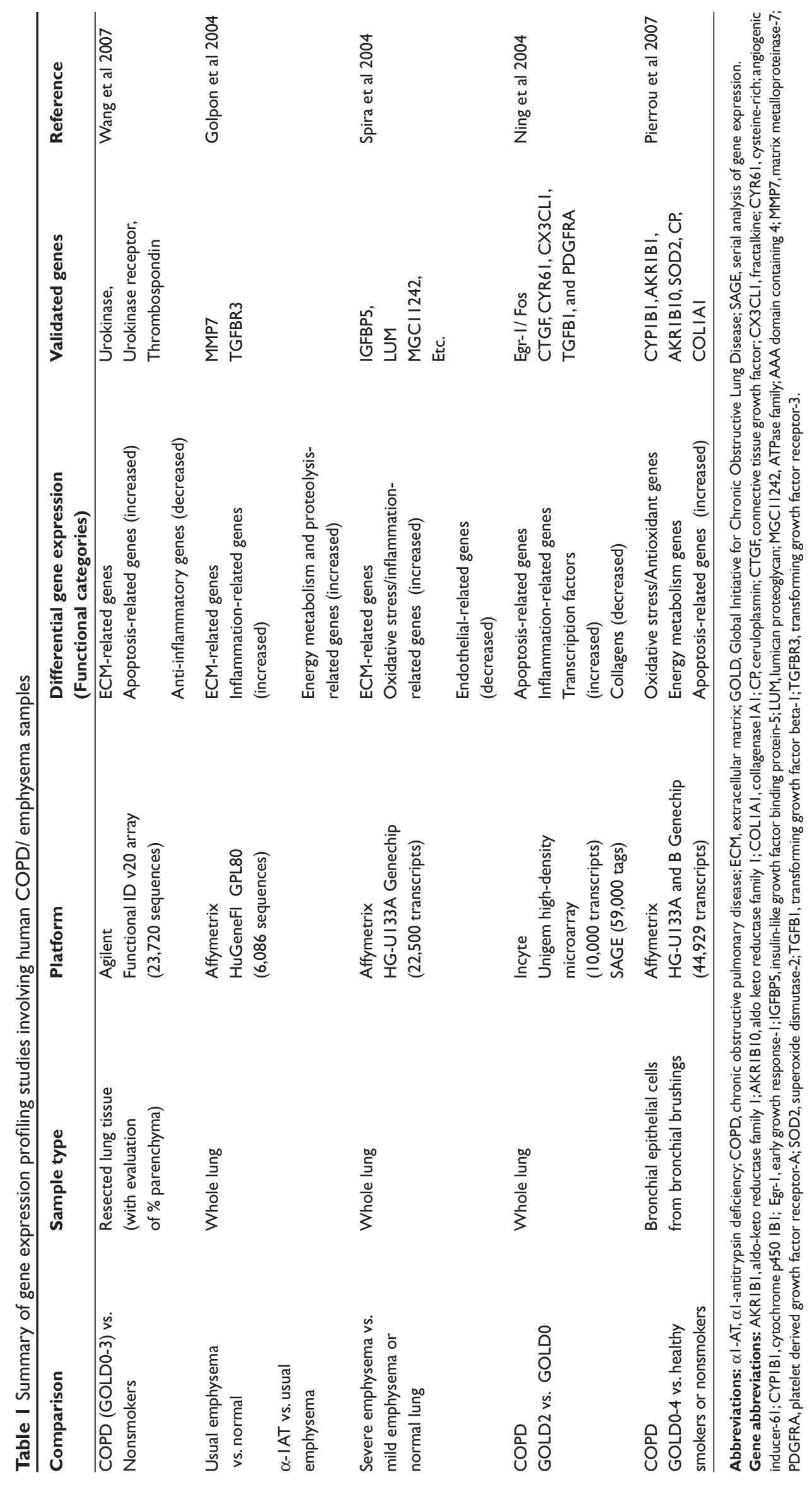


content can dramatically influence gene expression results. In addition to sample type and composition, variations in sample acquisition techniques, degree of disease severity, exclusion criteria, and microarray platforms may account in part for these differences (Wang et al 2008).

In animal models of smoke exposure, a recent study has compared the gene expression profiles of rat lungs exposed to acute and chronic cigarette smoking (Stevenson et al 2007). Rats were exposed to cigarette smoke for various intervals up to 8 months. This study identified an early acute phase of differential gene expression associated with initial responses to smoke exposure, followed by a chronic phase of gene expression associated with the development of pathological changes resembling COPD, including neutrophil influx, inflammation and emphysematous changes. Among the early differentially expressed genes in response to acute smoke exposure included several associated with major metabolic pathways including glycolysis and oxidative phosphorylation. The early acute phase displayed upregulation of stress proteins such as heme oxygenase-1 (HO-1), thioredoxin, manganese superoxide dismutase (MnSOD) and detoxification related genes, as well as a major transcriptional regulator associated with acute stress responses, NF-E2 related factor-2, (Nrf-2). These results were indicative of increased energy demand and adaptive responses of pulmonary cells exposed to acute smoke. The early chronic phase (2-3 weeks) was associated with increased expression of ECM related proteases (ie, MMP-12), cytokines, chemokines, and stress response proteins. Persistent activation of stress proteins was also observed in rats acutely exposed to smoke followed by smoking cessation. The late chronic phase ( 8 months) was associated with major alterations in genes involved in immune regulation (Stevenson et al 2007).

Collectively, these human and animal gene expression profiling studies have suggested several novel candidate therapeutic targets, as outlined in the following sections.

\section{Apoptosis/cell cycle arrest}

The lung consists of a heterogenous population of diverse cell types. These include epithelial cells which form the lining of the airway, bronchii, and alveoli, interstitial fibroblasts which provide lung structure, endothelial cells which line the pulmonary vasculature; and smooth muscle cells that provide the contractility of the airway and pulmonary vasculature. Furthermore the lung contains resident inflammatory cells such as alveolar macrophages.

Recent studies of lung tissues from COPD patients have revealed the presence of apoptotic cells in greater numbers than in control lungs or those from smokers without COPD (Henson et al 2006). Among those cells which undergo apoptosis in situ include alveolar and bronchial epithelial cells as well as endothelial cells in the parenchyma. In rodents, induction of endothelial or epithelial cell apoptosis is accompanied by loss of pulmonary alveoli and pathological evidence of emphysematous changes (Kasahara et al 2000). Blockade of the vascular endothelial growth factor receptors caused epithelial cell apoptosis, oxidative stress and emphysema-like disease in rats and mice (Petrache et al 2005). In addition, lung fibroblasts from patients with emphysema show a reduced proliferation rate (Nobukuni et al 2002). Cigarette smoke induces cellular senescence and cell cycle arrest in lung fibroblasts (Nyunoya et al 2006). This evidence suggests that apoptosis and cell cycle arrest may play crucial roles in emphysema and COPD. Gene expression profiling in emphysema revealed that many apoptosis/cell cycle-related genes were tightly correlated with lung function as assessed by the forced expiratory volume in one second $\left(\mathrm{FEV}_{1}\right)($ Spira et al 2004). The gene expression profiling studies of Ning and colleagues (2004) observed increased expression of apoptosis related factors and of inhibitors of cellular proliferation (eg, CDKN1A and CDC2L1) in GOLD2 COPD lung relative to GOLD0 samples, and furthermore, that mitochondrial function-related genes were significantly downregulated in COPD lungs. Collectively, these studies suggest that inhibition of smoking-induced apoptosis/cell cycle arrest may have a therapeutic value in COPD prevention. Although therapeutic agents targeting apoptosis pathways are currently starting to emerge in biomedical research and human clinical trials (eg, for cancer therapy), we have yet to observe the translational application of these targets in COPD. However, with improved understanding in the mechanism of smoke induced cell death and apoptosis of critical lung cells important in tissue repair and remodeling, one could envision how anti-apoptotic agents could mitigate lung epithelium cell death in susceptible smokers with epithelial cell injury and cell death.

\section{Inflammation}

Chronic inflammation has been well recognized as a primary characteristic of COPD. Inhibition of the inflammatory response may represent an important therapeutic strategy for COPD. Gene expression profiling has identified a number of inflammation-related proteins that are modulated in COPD, including adhesion molecules, as well as signaling molecules and transcription factors known to regulate pro-inflammatory processes, as outlined below. Anti-inflammatory drugs 
directed against these molecular targets are currently under development.

\section{Adhesion molecules}

Recruitment and activation of inflammatory cells is an important event in the pathogenesis of COPD. The adherence of neutrophils, monocytes, and cytotoxic T-cells to the vascular wall and subsequent recruitment into the lungs and respiratory tract is dependent on adhesion molecules expressed by these cells and on endothelial cells in the pulmonary and bronchial vasculatures (Barnes 2005). The expression of macrophage associated antigen-1 (Mac-1) is increased on neutrophils of patients with stable COPD, suggesting that targeting such adhesion molecules may have therapeutic potential (Noguera et al 1998). The expression of leukocyte as well as epithelial/endothelial surface adhesion molecules was elevated in resected lung tissue from heavy smokers, consistent with an inflammatory response to lung inflammation but not correlated to the degree of airways obstruction (Gonzalez et al 1996). Increased levels of circulating intercellular adhesion molecule-1 (cICAM-1) and circulating E-selectin (c-E-selectin) in serum, and of cICAM-1 in BAL, were observed in COPD patients relative to controls. The serum levels of c-E-selectin correlated significantly with lung function (Riise 1994). Gene expression profiling studies have indicated that adhesion molecules occur among the classes of genes that are up-regulated in the total lung tissue of COPD patients compared with healthy smokers (Ning et al 2004).

Selectin-mediated adhesion of leukocytes to the vascular endothelium is a key early event in the initiation of the inflammatory response, and therefore selectin may represent a valid target for therapeutic intervention (Romano 2005). Several adhesion molecules can now be inhibited pharmacologically, and among these inhibitors, bimosiamose is the leading selectin inhibitor in clinical development (Davenpeck et al 2000; Romano 2005).

\section{Cytokines and chemokines}

The inflammatory response associated with COPD involves multiple cytokines which regulate the activation, proliferation, and differentiation of inflammatory cells. The recruitment of inflammatory cells to the lung including neutrophils, monocytes, eosinophils, and $\mathrm{CD}^{+}$cytotoxic T-cells, is orchestrated by chemokines that are released from alveolar macrophages and pulmonary epithelial cells. In exhaled breath condensate, healthy smokers displayed increased levels of interleukins (IL), such as IL-1 $\beta$, IL-6, IL-8, IL-10 and IL-12p70, and tumor necrosis factor alpha (TNF- $\alpha$ ) when compared with nonsmokers, and all cytokines were increased on COPD exacerbations when compared with patients with stable COPD (Gessner et al 2005). TNF- $\alpha$ was increased in smoker's serum and sputum samples (Keatings et al 1996). The neutrophil chemokines IL-8 (CXCL8) and growth related oncogene-alpha (GRO $\alpha)$, as well as the monocyte chemotactic protein-1 (MCP-1) were also increased in sputum from COPD patients (Yamamoto et al 1997; Traves et al 2002) and negatively correlated with lung function. In agreement with these observations, gene expression profiling revealed that many inflammatory cytokine (ie, TNF- $\alpha$ receptors and interleukin receptors), and chemokine-related genes (ie, fractalkine/CX3CL1) were significantly increased in COPD patients (Ning et al 2004).

Strategies involving modulation of cytokine or cytokine receptor gene expression may have future potential for COPD therapy, as suggested by recent experiments in animal models. For example, transgenic overexpression of TNF- $\alpha$ led to emphysema and exaggerated alveolar inflammation (Fujita et al 2001), whereas TNF- $\alpha$ receptor knockout mice showed significant protection against cigarette smoke induced emphysema (Churg et al 2004). The IL-1 receptor/ TNF- $\alpha$ receptor double knockout mice were protected against elastase-induced emphysema (Lucey et al 2002).

Chemokine receptor blockade using small molecule antagonists has also been proposed as an anti-inflammatory strategy with potential applications in COPD (Donnelly and Barnes 2006). The chemokine receptors implicated in COPD, include CXCR1 and CXCR2 which are expressed on monocytes and neutrophils; CXCR3, which is expressed on T-cell and B-cells; and receptors of the CCR series $(-1,-2,-3$, $-5)$ which are expressed on various leukocytes (Donnelly and Barnes 2006). Several CXCR2 antagonists have been shown to reduce neutrophil influx in animal models in response to proinflammatory stimuli or cigarette smoke exposure (Hay and Sarau 2001; Stevenson et al 2005; Thatcher et al 2005). Recent human clinical studies have shown promise for CCR1 antagonists in reducing inflammation associated with rheumatoid arthritis (Haringman 2003). Future investigations and clinical trials have yet to reveal the effectiveness of this class of pharmaceuticals in human COPD (Barnes 2005).

\section{$\mathrm{NF \kappa B}$}

The expression of many of chemokines such as IL-8/CXCL8 and cytokines such as TNF $\alpha$ is regulated by transcription factor, $\mathrm{NF} \kappa \mathrm{B}$, a pleiotropic regulator of inflammatory processes (Figure 2) (Yagi et al 2006; Yang et al 2006). NFkB is in mac- 


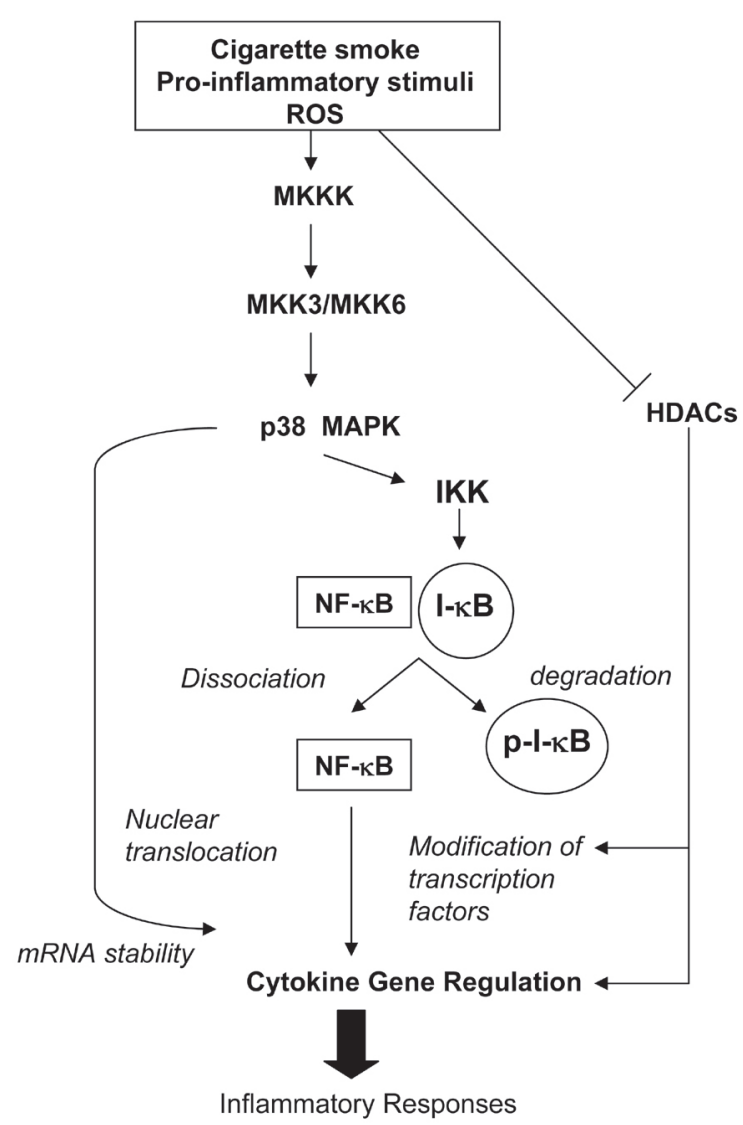

Figure 2 Epigenetic factors involved in the regulation of inflammation.

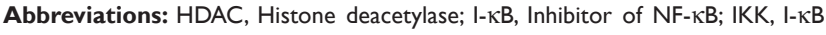
kinase; MKKK, mitogen-activated protein kinase-3; MKK3/6, mitogen-activated protein

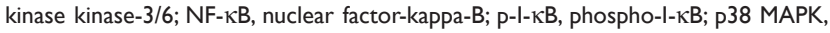
p38 mitogen-activated protein kinase; ROS, reactive oxygen species.

rophages and epithelial cells of COPD patients (Di Stefano et al 2002; Caramori et al 2003). Szulkaowski and colleagues (2006) examined the proteosomal degradation of IкB $\alpha$ and NFKB DNA binding activity in the human lung in response to cigarette smoking. I $\mathrm{\kappa} \mathrm{B} \alpha$ levels were significantly decreased while NFKB DNA binding was significantly increased in healthy smokers and current smokers with moderate COPD, compared with healthy nonsmokers (Szulkaowski et al 2006). Analysis of transcription factor binding sites occurring in the promoter regions of differentially-expressed genes revealed that NFKB sties were overrepresented among those genes that were downregulated by smoking (relative to nonsmokers) and upregulated by COPD (relative to healthy smokers) (Pierrou et al 2007). These observations, taken together, suggest that modulation of NFKB activity may represent a potential target in COPD therapy. There are several approaches to inhibition

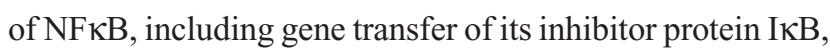
and small-molecule inhibitors of IKB-kinase (IKK) (Newton et al 2007).

\section{P38 MAPK}

A large number of inflammatory response-related signal transduction molecules are modulated in COPD patients, including mitogen-activated protein kinases (MAPK) and related proteins (Golpon et al 2004; Ning et al 2004). MAPK play an important role in chronic inflammation (Johnson et al 2002) (Figure 2). Indeed, activation of members of the MAPK family triggers the activation of several transcription factors, including NFKB. This eventually results in the expression of a battery of distinct genes that can regulate pro-inflammatory, pro-apoptotic and antiproliferative responses (Johnson 2002). Among these, the p38 MAPK pathway is activated by cellular stress and regulates the expression of inflammatory cytokines, including IL-8, TNF $\alpha$, and matrix metalloproteases (MMPs) (Meja 2000). Inhibitors of the p38 MAPK family have been shown to exert anti-inflammatory effects in preclinical disease models, primarily through the inhibition of the expression of inflammatory mediators (Lee et al 2005). Several promising compounds have also progressed to clinical trials. One of these, SB239063, can reduce neutrophil infiltration after inhaled endotoxin and lower the concentrations of IL- 6 and MMP9 in bronchoalveolar lavage fluid of rats, suggesting a potential role in COPD treatment (Underwood et al 2000).

\section{Histone deacetylases}

Many factors, including specific DNA sequences, histones, nonhistone chromosomal proteins, transcriptional activators/ repressors and the transcriptional machinery, are all necessary for the establishment of an active transcription complex. Histone acetytransferases (HAT) and histone deacetylase (HDAC) are families of enzymes that regulate chromatin structure and thereby affect inflammatory gene expression (Barnes et al 2005). Acetylation of core histones by coactivator proteins that possess intrinsic HAT activity leads to unwinding of chromatin, which subsequently allows transcription factors and RNA polymerase II to activate gene transcription. Conversely, deacetylation of core histones by HDAC is generally associated with transcriptional repression.

Recent evidence has suggested an important role for HDAC activity in COPD. Surgically resected lung tissue from nonsmokers or GOLD0-4 smokers, showed significant decreases of HDAC activity in COPD lung tissue relative to nonsmokers whose magnitude correlated with disease severity and inflammatory gene expression (Ito et al 2005). In addition to lung tissue, HDAC activity was also decreased in alveolar macrophages from BAL samples of COPD patients (Ito et al 2005). Furthermore, the expression of HDAC2 
mRNA and protein in peripheral lung tissue and BAL macrophages, declined as a function of disease progression (Ito et al 2005).

Cells exposed to cigarette smoke extract (CSE) also exhibit decreased HDAC activity and protein levels, and this process appears to be redox-dependent (Yang et al 2006). In rats exposed to cigarette smoke, HDAC2 activity and protein expression significantly decreased at 3 days of treatment, while histone-3 phospho-acetylation and histone-4 acetylation increased at 8 weeks of treatment (Marwick et al 2004). Consistent with these findings, our gene profiling results also revealed that certain histones were increased in COPD and that HDAC2 was significantly decreased (Ning et al 2004). All these findings could explain therapeutic refractoriness and perpetuation of HDAC and could reveal an evolving therapeutic target in COPD. For example, theophylline restores histone deacetylase activity and steroid responses in COPD macrophages, suggesting potential application of this drug in COPD therapy (Cosio et al 2004).

\section{Egr-I}

Among the thousands of genes differentially expressed in COPD, Egr-1 mRNA is one of the significantly up-regulated transcripts identified by both SAGE and microarray analysis with confirmation by RT-PCR and immunohistochemical approaches (Ning et al 2004). Egr-1 is a zinc finger transcription factor classified as an immediate-early response protein, which is potentially induced by a variety of cellular stressors (Lee et al 1996). As a major transcription factor, Egr-1 regulates the expression of a large number of genes, including repair enzyme systems, angiogenic factors, cytokines, apoptotic factors, cell cycle factors, metabolic factors and proteases (Yan et al 2000). Ning et al observed that exposure to aqueous cigarette smoke extract (CSE) treatment could stimulate Egr-1 expression and transcriptional activity in human lung fibroblasts. In addition, matrix metalloproteinase (MMP)-2 and MMP-9 activities were modulated in an Egr-1dependent manner in mouse lung fibroblast cells treated with cytomix (Ning et al 2004). These data suggest that Egr-1 may serve as a potentially important molecule, which could play a key role in development of cigarette smoke-related COPD by regulating MMP activity and subsequently accelerating the turnover of ECM proteins.

\section{Protease/antiprotease imbalance}

A central hypothesis for the development of emphysema is that various proteases break down connective tissue components in lung parenchyma (Barnes et al 2003). Increased proteolysis in the lung leads to aberrant remodeling and/or degradation of the ECM. Thus, altered ECM deposition contributing to airway wall remodeling is an important feature of asthma and COPD. A large number of ECM-related genes were up-regulated in the lungs of smokers with emphysema, and those were correlated to the $\mathrm{FEV}_{1}$ (Spira et al 2004). Inhibition of these proteolytic enzymes or an increase in endogenous antiproteinases should therefore be beneficial and theoretically should prevent the progression of airflow obstruction in COPD. Among those proteases, neutrophil elastase, MMPs, and cathepsins are the most studied candidates that account for the protease/antiprotease imbalance in COPD.

The evidence in support of a significant role of neutrophil elastase in the pathogenesis of emphysema has relied on the finding of early emphysema in patients deficient in $\alpha 1$-antitrypsin, the major inhibitor of neutrophil elastase. Emphysema caused by lung instillation of porcine pancreatic elastase has been a disease model widely used in support of the protease/antiprotease imbalance over the past 30 years (Yoshida et al 2007). Neutrophil elastase is a neutral serine protease and is a major constituent of lung elastolytic activity. It also potently stimulates the secretion of mucus and induces IL-8 release from epithelial cells and therefore may perpetuate the inflammatory stage. Neutrophil elastase null mice were significantly protected against chronic cigarette smoke induced emphysema, which was associated with decreased MMP-12 activation and increased tissue inhibitor of matrix metalloproteinase activity (Shapiro et al 2003). Several drugs have been shown to inhibit neutrophil elastase-induced lung injury in experimental animals, and to inhibit neutrophil elastase-induced secretion of mucus in vitro (Williams et al 1991; Ohbayashi 2002).

There is substantial evidence of increased expression of several MMPs in emphysematous lungs. MMPs are a family of proteolytic enzymes that have a number of important physiological roles including remodeling of the extracellular matrix, facilitating cell migration, cleaving cytokines, and activating defensins. However, excess MMP activity may lead to tissue destruction. MMPs have been suggested as the major proteolytic enzymes involved in the pathogeneses of COPD because these proteins are a unique family of metalloenzymes that, once activated, can destroy connective tissue. MMP-12 activation has been a leading candidate proteinase responsible for pulmonary emphysema, because it is predominantly produced by alveolar macrophages and can degrade elastin. There is abundant evidence linking MMP-12 and experimental emphysema (Yoshida et al 2007). MMP-12 
null mice are protected from the development of cigarette smoke induced emphysema (Hautamaki et al 1997). Recent data indicate that the use of selective inhibitors of MMPs might lead to new therapies for acute and chronic inflammatory diseases (Cataldo et al 2003; Whelan 2004).

Gene expression profiling also revealed that multiple cathepsins were increased in COPD lung tissues (Ning et al 2004; Spira et al 2004). Cathepsin G has elastolytic activity. Cathepsins B, L, and S are also released from macrophages. In cigarette smoke-exposed mice, cathepsin D was highly expressed in pulmonary macrophages and dendritic cells (Bracke 2005). Inducible targeting of IL-13 to the adult lung causes matrix metalloproteinase- and cathepsin-dependent emphysema (Zheng et al 2000). These findings suggest that cathepsin inhibitors might be a useful approach for COPD therapy.

\section{Fibrosis}

While apoptosis is implicated in epithelial cell death and alveolar destruction leading to emphysema, increased fibroblast proliferation may account for small airways disease also present in COPD (Yoshida and Tuder 2007). Recent studies have shown that fibrosis and COPD can coexist in the same patient (Gauldie et al 2006). The transforming growth factor (TGF)- $\beta 1$ is highly expressed in epithelium and macrophages of small airways of smokers with COPD (de Boer et al 1998; Takizawa et al 2001), suggesting a role for TGF- $\beta 1$ in the development of COPD. TGF- $\beta 1$ is a pleiotropic cytokine with a wide variety of effects on cellular proliferation, differentiation, and inflammation. TGF- $\beta 1$ may be important in inducing the fibrosis and narrowing of peripheral airways (obstructive bronchiolitis) in COPD. Gene expression profiling revealed that TGF- $\beta 1$ and its regulatory pathways were significantly increased in COPD patients (Ning et al 2004; Wang et al 2008). Thus, inhibition of TGF- $\beta 1$ signaling may be a useful therapeutic strategy in COPD. Small molecule antagonists that inhibit TGF- $\beta 1$-receptor kinase are now under development (Ishikawa et al 2003; Akhurst 2006).

\section{Oxidative stress}

Cigarette smoke is a rich source of oxidants. Accumulating evidence has shown increased oxidative stress in smokers (Pierrou et al 2007), as revealed by the increased concentrations of $\mathrm{H}_{2} \mathrm{O}_{2}$ in exhaled breath (Horvath et al 2004), and other markers including lipid peroxidation end-products (Lapenna et al 1995), oxidatively modified protein (Pignatelli et al 2001), and DNA damage (Cuzick et al 1990). In COPD patients, exhaled biomarkers of lipid peroxidation such as 8-isoprostane (Biernacki 2003) and ethane (Paredi et al 2000) are significantly increased. Gene profiling studies also show that the expression of redox/stress related genes are tightly correlated with the $\mathrm{FEV}_{1}$ and carbon monoxide lung diffusion capacity $\left(\mathrm{DL}_{\mathrm{CO}}\right)$ (Spira et al 2004), and that several major antioxidant enzymes such as glutaredoxin and catalase are decreased in COPD by 5 -fold compared with healthy smokers (Ning et al 2004). Genetic ablation of Nrf2, a pivotal transcriptional factor regulating the expression of many antioxidant enzymes, enhances susceptibility to cigarette smoke-induced emphysema (Rangasamy et al 2004), and severe airway inflammation and asthma in mice (Rangasamy et al 2005). Analysis of transcription factor binding sites occurring in the promoter regions of differentially expressed genes revealed that Nrf2 sties were overrepresented among those genes that were both upregulated by smoking (relative to nonsmokers) and upregulated by COPD (relative to healthy smokers) (Pierrou et al 2007). Enhancement of cellular antioxidant capacity has been suggested as a possible target for COPD therapy. Effective antioxidants including stable glutathione analogs, superoxide dismutase mimics, and selenium-based drugs, are now in development for clinical use (MacNee 2000).

\section{Heme oxygenase- I}

Heme oxygenase-1 (HO-1) is a major inducible stress protein of mammalian cells and tissues. HO-1 expression confers cytoprotection in a variety of tissue injury and disease models (Ryter et al 2006), among which include experimental elastase-induced emphysema (Shinohara et al 2005). Recent studies have revealed that HO-1 is highly induced by cigarette smoke exposure protocols, including mainstream smoke, cigarette smoke condensate, and aqueous cigarette smoke extract (CSE) in a number of cell types (Knorr-Wittmann et al 2005; Fukano et al 2006a, 2006b; Palozza et al 2006). We have shown that CSE induces HO-1 in bronchial and other pulmonary epithelial cell types exposed to CSE in vitro, as well as in rat lung tissue exposed to chronic cigarette smoke inhalation (Slebos et al 2007). Elevated levels of HO-1 were reported in the alveolar spaces of chronic smokers with and without COPD relative to nonsmokers (Maestrelli et al 2001). Paradoxically, gene expression profiling revealed that HO-1 gene expression was downregulated in lung tissue from end stage COPD relative to healthy smokers (Ning et al 2004). This is consistent with reports that have shown decreased HO-1 expression in alveolar macrophages of severe COPD patients relative to those of smokers without lung function impairment (Maestrelli 2003). Furthermore, 
ex-smoking COPD patients were reported to have reduced HO-1 expression in alveolar macrophages relative to healthy ex-smokers (Slebos et al 2004). These preliminary observations indicate that stress protein responses such as HO-1 may be impaired during COPD progression, which may contribute to the etiology of this disease.

Recent studies have described a potential genetic basis for the HO-1 downregulation observed in COPD. A microsatellite $(\mathrm{GT})_{\mathrm{n}}$ dinucleotide length polymorphism can occur in the promoter region of the ho- 1 gene, resulting in a lower production of HO-1 in individuals that carry the long (L) allele $[(\mathrm{GT}) n \geq 30]$ of this polymorphism (Yamada et al 2000). This polymorphism was linked with the development of COPD in a cohort of Japanese men (Yamada et al 2000). In a retrospective study of French smokers the $L$ allele $[(\mathrm{GT}) \mathrm{n} \geq 33]$ was associated with decreased lung function parameters relative to noncarriers. The greatest decline in lung function was observed in heavy smokers that carried the L allele (Guenegou et al 2006). These observations suggest that a genetically-dependent impairment of HO-1 expression may occur in subpopulations, possibly linked to increased susceptibility to smoke-induced oxidative stress (Yamada et al 2000).

\section{Concluding remarks}

Several important limitations to gene expression analyses using microarrays must be considered. For example, the number of lung tissue samples used for the assays is typically relatively small, which makes certain results based on one study not truly representative. Furthermore, the up or downregulation of specific genes may be different or contradictory between studies. Additional studies on larger numbers of lung tissue samples are needed to validate the current findings and to address the variability in the cellular composition of the tissues, given the heterogeneous nature of disease. More studies are also needed to determine the functions of these significantly changed genes in the pathogenesis of COPD, and to establish whether DNA sequence variation within these genes causes or predicts COPD.

Gene expression profiling analyses (eg, microarray) to identify candidate genes important in the pathogenesis of COPD represent tour de force approaches in the current genomics era to better understand the pathogenesis of human disease such as COPD. However, we are just at the tip of the "iceberg" in that evolving state of the art approaches such as proteomics, metabolomics and gene polymorphism studies add to the total genomic armamentarium to better study the pathogenesis of COPD. Furthermore, laser capture microscopy of specific cell types in the lung can be used to obtain gene expression or protein data at a cellular level. Noninvasive methods to obtain human lung cells including exhaled breath condensate or sputum will provide exciting opportunities in the future. These approaches not only provide molecular signatures of COPD but also can serve as biomarkers to help in the diagnosis, treatment, outcome and response to therapy for COPD patients.

\section{Acknowledgments}

This work was supported in part by awards from the American Heart Association to S. W. Ryter (AHA \#0335035N), and H.P. Kim (AHA \#0525552U), and NIH grants R01-HL60234, R01-HL55330, R01-HL079904, and P01-HL 70807 awarded to A. M. K. Choi. The authors have no conflicts of interest to declare.

\section{References}

Akhurst RJ. 2006. Large- and small-molecule inhibitors of transforming growth factor-beta signaling. Curr Opin Investig Drugs, 7:513-21.

Barnes PJ, Adcock IM, Ito K. 2005. Histone acetylation and deacetylation: importance in inflammatory lung diseases. Eur Respir J, 25:552-63.

Barnes PJ, Hansel TT. 2004. Prospects for new drugs for chronic obstructive pulmonary disease. Lancet, 364:985-96.

Barnes PJ, Shapiro SD, Pauwels RA. 2003. Chronic obstructive pulmonary disease: molecular and cellular mechanisms. Eur Respir $J, 22: 672-88$.

Barnes PJ. 1999. Novel approaches and targets for treatment of chronic obstructive pulmonary disease. Am J Respir Crit Care Med, 160:S72-79.

Barnes PJ. 2004. COPD: is there light at the end of tunnel? Curr Opin Pharmacol, 4:263-72.

Barnes PJ. 2005. New approaches to COPD. Eur Respir Res, 14: 2-11.

Barnes PJ, Stockley, RA. 2005. COPD: current therapeutic interventions and future approaches. Eur Respir J, 25:1084-106.

Biernacki WA, Kharitonov SA, Barnes PJ. 2003. Increased leukotriene B4 and 8-isoprostane in exhaled breath condensate of patients with exacerbations of COPD. Thorax, 58:294-98.

Bracke K, Cataldo D, Maes T, et al. 2005. Matrix metalloproteinase-12 and cathepsin D expression in pulmonary macrophages and dendritic cells of cigarette smoke-exposed mice. Int Arch Allergy Immunol, 138:169-79.

Caramori G, Romagnoli M, Casolari P, et al. 2003. Nuclear localisation of p65 in sputum macrophages but not in sputum neutrophils during COPD exacerbations. Thorax, 58:348-51.

Cataldo DD, Gueders MM, Rocks N, et al. 2003. Pathogenic role of matrix metalloproteases and their inhibitors in asthma and chronic obstructive pulmonary disease and therapeutic relevance of matrix metalloproteases inhibitors. Cell Mol Biol (Noisy-le-grand), 49:875-84.

Churg A, Wang RD, Tai H, et al. 2004. Tumor necrosis factor-alpha drives $70 \%$ of cigarette smoke-induced emphysema in the mouse. Am J Physiol Lung Cell Mol Physiol, 290:419-28.

Cosio BG, Tsaprouni L, Ito K, et al. 2004. Theophylline restores histone deacetylase activity and steroid responses in COPD macrophages. J Exp Med, 200:689-95.

Cuzick J, Routledge MN, Jenkins D, et al. 1990. DNA adducts in different tissues of smokers and non-smokers. Int J Cancer, 45:673-78.

Davenpeck KL, Berens KL, Dixon RA, et al. 2000. Inhibition of adhesion of human neutrophils and eosinophils to P-selectin by the sialyl Lewis antagonist TBC1269: preferential activity against neutrophil adhesion in vitro. J Allergy Clin Immunol, 105:769-75. 
de Boer WI, van Schadewijk A, Sont JK, et al. 1998. Transforming growth factor beta 1 and recruitment of macrophages and mast cells in airways in chronic obstructive pulmonary disease. Am J Respir Crit Care Med, 158:1951-57.

Di Stefano A, Caramori G, Capelli A, et al. 2002. Increased expression of $\mathrm{NF}-\mathrm{\kappa B}$ in bronchial biopsies from smokers and patients with COPD. Eur Respir J, 20:556-63.

Donnelly LE, and Barnes PJ. 2006. Chemokine receptors as therapeutic targets in chronic obstructive pulmonary disease. Trends Pharmacol Sci, 27:546-52.

Fujita M, Shannon JM, Irvin CG, et al. 2001. Overexpression of tumor necrosis factor-alpha produces an increase in lung volumes and pulmonary hypertension. Am J Physiol Lung Cell Mol Physiol, 280:L39-L49.

Fukano Y, Oishi M, Chibana F, et al. 2006a. Analysis of the expression of heme oxygenase-1 gene in human alveolar epithelial cells exposed to cigarette smoke condensate. J Toxicol Sci, 31:99-109.

Fukano Y, Yoshimura H, Yoshida T. 2006b. Heme oxygenase-1 gene expression in human alveolar epithelial cells (A549) following exposure to whole cigarette smoke on a direct in vitro exposure system. Exp Toxicol Pathol, 57:411-8.

Gauldie J, Kolb M, Ask K, et al. 2006. Smad3 signaling involved in pulmonary fibrosis and emphysema. Proc Am Thorac Soc, 3:696-702.

Gessner C, Scheibe R, Wotzel M, et al. 2005. Exhaled breath condensate cytokine patterns in chronic obstructive pulmonary disease. Respir Med, 99:1229-40.

Golpon HA, Coldren CD, Zamora MR, et al. 2004. Emphysema lung tissue gene expression profiling. Am J Respir Cell Mol Biol, 31:595-600.

Gonzalez S, Hards J, van Eeden S, et al. 1996. The expression of adhesion molecules in cigarette smoke-induced airways obstruction. Eur Respir $J, 9: 1995-2001$.

Guenegou A, Leynaert B, Benessiano J, et al. 2006. Association of lung function decline with the heme oxygenase- 1 gene promoter microsatellite polymorphism in a general population sample. Results from the European Community Respiratory Health Survey (ECRHS), France. $J$ Med Genet, 43:e43.

Haringman JJ, Kraan MC, Smeets TJ, et al. 2003. Chemokine blockade and chronic inflammatory disease: proof of concept in patients with rheumatoid arthritis. Ann Rheum Dis, 6:715-21.

Hautamaki RD, Kobayashi DK, Senior RM, et al. 1997. Requirement for macrophage elastase for cigarette smoke induced emphysema in mice. Science, 277:2002-4.

Hay DW, Sarau HM. 2001. Interleukin-8 receptor antagonists in pulmonary diseases. Curr Opin Pharmacol, 1:242-7.

Henson PM, Vandivier RW, Douglas IS. 2006. Cell death, remodeling, and repair in chronic obstructive pulmonary disease? Proc Am Thorac Soc, 3:713-7.

Horvath I, Donnelly LE, Kiss A, et al. 2004. Exhaled nitric oxide and hydrogen peroxide concentrations in asthmatic smokers. Respiration, $71: 463-8$.

Ishikawa T, Kume H, Kondo M, et al. 2003. Inhibitory effects of interferongamma on the heterologous desensitization of beta-adrenoceptors by transforming growth factor-beta 1 in tracheal smooth muscle. Clin Exp Allergy, 33:808-15.

Ito K, Ito M, Elliott WM, et al. 2005. Decreased histone deacetylase activity in chronic obstructive pulmonary disease. N Engl J Med, 352:1967-76.

Johnson GL, Lapadat R. 2002. Mitogen-activated protein kinase pathways mediated by ERK, JNK, and p38 protein kinases. Science, 298:1911-2.

Kasahara Y, Tuder RM, Taraseviciene-Stewart L, et al. 2000. Inhibition of VEGF receptors causes lung cell apoptosis and emphysema. J Clin Invest, 106:1311-9.

Keatings VM, Collins PD, Scott DM, et al. 1996. Differences in interleukin-8 and tumor necrosis factor-alpha in induced sputum from patients with chronic obstructive pulmonary disease or asthma. Am J Respir Crit Care Med, 153:530-4.
Knorr-Wittmann C, Hengstermann A, Gebel S, et al. 2005. Characterization of Nrf2 activation and heme oxygenase-1 expression in NIH3T3 cells exposed to aqueous extracts of cigarette smoke. Free Radic Biol Med, 39:1438-48

Lapenna D, Mezzetti A, de Gioia S, et al. 1995. Plasma copper and lipid peroxidation in cigarette smokers. Free Radic Biol Med, 19:849-52.

Lee MR, Dominguez C. 2005. MAP kinase p38 inhibitors: clinical results and an intimate look at their interactions with p38alpha protein. Curr Med Chem, 12:2979-94.

Lee SL, Sadovsky Y, Swirnoff AH, et al. 1996. Luteinizing hormone deficiency and female infertility in mice lacking the transcription factor NGFI-A (Egr-1). Science, 273:1219-21.

Lopez AD, Murray CC. 1998. The global burden of disease, 1990-2020. Nat Med, 4:1241-3.

Lucey EC, Keane J, Kuang PP, et al. 2002. Severity of elastase-induced emphysema is decreased in tumor necrosis factor-alpha and interleukin1 beta receptor-deficient mice. Lab Invest, 82:79-85.

Macnee W. 2000. Oxidants/antioxidants and COPD. Chest, 117:S303-317.

Maestrelli P, El Messlemani AH, De Fina O, et al. 2001. Increased expression of heme oxygenase (HO)-1 in alveolar spaces and $\mathrm{HO}-2$ in alveolar walls of smokers. Am J Respir Crit Care Med, 164:1508-13.

Maestrelli P, Paska C, Saetta M, et al. 2003. Decreased haem oxygenase1 and increased inducible nitric oxide synthase in the lung of severe COPD patients. Eur Respir J, 21:971-76.

Mayer AS, and Newman LS. 2001. Genetic and environmental modulation of chronic obstructive pulmonary disease. Respir Physiol, 128:3-11.

Meja KK, Seldon PM, Nasuhara Y, et al. 2000. p38 MAP kinase and MKK-1 co-operate in the generation of GM-CSF from LPS-stimulated human monocytes by an NF-KB-independent mechanism. Br J Pharmacol, 131:1143-53.

Newton R, Holden NS, Catley MC, et al. 2007. Repression of inflammatory gene expression in human pulmonary epithelial cells by small-molecule IkappaB kinase inhibitors. J Pharmacol Exp Ther, 321:734-42.

Ning W, Li CJ, Kaminski N, et al. 2004. Comprehensive gene expression profiles reveal pathways related to the pathogenesis of chronic obstructive pulmonary disease. Proc Natl Acad Sci USA, 101:14895-900.

Nobukuni S, Watanabe K, Inoue J, et al. 2002. Cigarette smoke inhibits the growth of lung fibroblasts from patients with pulmonary emphysema. Respir, 7:217-23.

Noguera A, Busquets X, Sauleda J, et al. Expression of adhesion molecules and $\mathrm{G}$ proteins in circulating neutrophils in chronic obstructive pulmonary disease. Am J Respir Crit Care Med, 158:1664-68.

Nyunoya T, Monick MM, Klingelhutz A, et al. 2000. Cigarette smoke induces cellular senescence. Am J Respir Cell Mol Biol, 35:681-88.

Ohbayashi H. 2002. Novel neutrophil elastase inhibitors as a treatment for neutrophil-predominant inflammatory lung diseases. Drugs, 5:910-23.

Palozza P, Serini S, Curro D, et al. 2006. beta-Carotene and cigarette smoke condensate regulate heme oxygenase-1 and its repressor factor Bach1: relationship with cell growth. Antioxid Redox Signal, 8:1069-80.

Paredi P, Kharitonov SA, Leak D, et al. 2000. Exhaled ethane, a marker of lipid peroxidation, is elevated in chronic obstructive pulmonary disease. Am J Respir Crit Care Med, 162:369-73.

Pauwels RA, Buist AS, Calverley PM, et al. 2001. Global strategy for the diagnosis, management, and prevention of chronic obstructive pulmonary disease. NHLBI/WHO Global Initiative for Chronic Obstructive Lung Disease (GOLD) Workshop summary. Am J Respir Crit Care Med, 163:1256-76.

Petrache I, Natarajan V, Zhen L, et al. 2005. Ceramide upregulation causes pulmonary cell apoptosis and emphysema-like disease in mice. Nat Med, 11:491-98.

Pierrou S, Broberg P, O’Donnell RA, et al. 2007. Expression of genes involved in oxidative stress responses in airway epithelial cells of smokers with chronic obstructive pulmonary disease. Am J Respir Crit Care Med, 175:577-86.

Pignatelli B, Li CQ, Boffetta P, et al. 2001. Nitrated and oxidized plasma proteins in smokers and lung cancer patients. Cancer Res, 61:778-84. 
Riise GC, Larsson S, Löfdahl CG, et al. 1994. Circulating cell adhesion molecules in bronchial lavage and serum in COPD patients with chronic bronchitis. Eur Respir J, 7:1673-7.

Rangasamy T, Cho CY, Thimmulappa RK, et al. 2004. Genetic ablation of Nrf2 enhances susceptibility to cigarette smoke-induced emphysema in mice. J Clin Invest, 114:1248-59.

Rangasamy T, Guo J, Mitzner WA, et al. 2005. Disruption of Nrf2 enhances susceptibility to severe airway inflammation and asthma in mice. $J$ Exp Med, 202:47-59.

Romano SJ. 2005. Selectin antagonists: therapeutic potential in asthma and COPD. Treat Respir Med, 4:85-94.

Ryter SW, Alam J, Choi AMK. 2006. Heme oxygenase/Carbon monoxide: From basic research to therapeutic applications. Physiol Rev, 86:583-50.

Shapiro SD, Goldstein NM, Houghton AM, et al. 2003. Neutrophil elastase contributes to cigarette smoke induced emphysema in mice. $\mathrm{Am} \mathrm{J}$ Pathol, 163:2329-35.

Shinohara T, Kaneko T, Nagashima Y, et al. 2005. Adenovirus-mediated transfer and overexpression of heme oxygenase 1 cDNA in lungs attenuates elastase-induced pulmonary emphysema in mice. Hum Gene Ther, 16:318-27.

Slebos DJ, Kerstjens HA, Rutgers SR, et al. 2004. Haem oxygenase-1 expression is diminished in alveolar macrophages of patients with COPD. Eur Respir J, 23:652-3.

Slebos DJ, Ryter SW, van der Toorn M, et al. 2007. Mitochondrial localization and function of heme oxygenase-1 in cigarette Smoke-induced cell death. Am J Respir Cell Mol Biol, 36:409-17.

Spira A, Beane J, Pinto-Plata V, et al. 2004. Gene expression profiling of human lung tissue from smokers with severe emphysema. Am J Respir Cell Mol Biol, 31:601-10.

Stevenson CS, Coote K, Webster R, et al. 2005. Characterization of cigarette smoke-induced inflammatory and mucus hypersecretory changes in rat lung and the role of CXCR2 ligands in mediating this effect. Am J Physiol Lung Cell Mol Physiol, 288:L514-22.

Stevenson CS, Docx C, Webster R, et al. 2007. Comprehensive gene expression profiling of rat lung reveals distinct acute and chronic responses to cigarette smoke inhalation. Am J Physiol Lung Cell Mol Physiol, 293:L1183-93.

Szulakowski P, Crowther AJL, Jimenez LA, et al. 2006. The effect of smoking on the transcriptional regulation of lung inflammation in patients with chronic obstructive pulmonary disease. Am J Respir Crit Care Med, 174:41-50.
Takizawa H, Tanaka M, Takami K, et al. 2001. Increased expression of transforming growth factor-beta1 in small airway epithelium from tobacco smokers and patients with chronic obstructive pulmonary disease (COPD). Am J Respir Crit Care Med, 163:1476-83.

Thatcher TH, McHugh NA, Egan RW, et al. 2005. Role of CXCR2 in cigarette smoke-induced lung inflammation. Am J Physiol Lung Cell Mol Physiol, 289:L322-8.

Traves SL, Culpitt SV, Russell RE, et al. 2002. Increased levels of the chemokines GROalpha and MCP-1 in sputum samples from patients with COPD. Thorax, 57:590-5

Underwood DC, Osborn RR, Bochnowicz S, et al. 2000. SB 239063, a p38 MAPK inhibitor, reduces neutrophilia, inflammatory cytokines, MMP-9, and fibrosis in lung. Am J Physiol Lung Cell Mol Physiol, 279:L895-902.

Wang IM, Stepaniants S, Boie Y, et al. 2008. Gene expression profiling in patients with chronic obstructive pulmonary disease and lung cancer. Am J Respir Crit Care Med, 177:402-11.

Whelan CJ. 2004. Metalloprotease inhibitors as anti-inflammatory agents: an evolving target? Curr Opin Investig Drugs, 5:511-6.

Williams JC, Falcone RC, Knee C, et al. 1991. Biologic characterization of ICI 200,800 and ICI 200,355, novel inhibitors of human neutrophil elastase. Am Rev Respir Dis, 144:875-83.

Yagi O, Aoshiba K, Nagai A, et al. 20006. Activation of nuclear factorkappaB in airway epithelial cells in patients with chronic obstructive pulmonary disease. Respir, 73:610-6.

Yamada N, Yamaya M, Okinaga S, et al. 2000. Microsatellite polymorphism in the heme oxygenase-1 gene promoter is associated with susceptibility to emphysema. Am J Hum Genet, 66:187-95.

Yamamoto C, Yoneda T, Yoshikawa M, et al. 1997. Airway inflammation in COPD assessed by sputum levels of interleukin-8. Chest, 112:505-10.

Yan SF, Pinsky DJ, Mackman N, et al. 2000. Egr-1: is it always immediate and early? J Clin Invest, 105:553-4.

Yang SR, Chida AS, Bauter MR, et al. 2006. Cigarette smoke induces proinflammatory cytokine release by activation of NF-kappaB and posttranslational modifications of histone deacetylase in macrophages. Am J Physiol Lung Cell Mol Physiol, 291:L46-57.

Yoshida T, Tuder RM, 2007. Pathobiology of cigarette smoke-induced chronic obstructive pulmonary disease. Physiol Rev, 87:1047-82.

Zheng T, Zhu Z, Wang Z, Homer RJ, et al. 2000. Inducible targeting of IL-13 to the adult lung causes matrix metalloproteinase- and cathepsindependent emphysema. J Clin Invest, 106:1081-93. 